\title{
Early Predictors of Mortality in Patients with COPD, in Relation to Respiratory and Non-Respiratory Causes of Death - A National Register Study
}

This article was published in the following Dove Press journal: International Journal of Chronic Obstructive Pulmonary Disease

Ingela Henoch (D) ${ }^{1,2}$

Ann Ekberg-Jansson ${ }^{3,4}$

Claes-Göran Löfdahl ${ }^{5,6}$

Peter Strang $\mathbb{D}^{7,8}$

'Department of Research and Development, Angered Local Hospital, Gothenburg, Sweden; ${ }^{2}$ Institute of Health and Care Sciences, Sahlgrenska Academy, University of Gothenburg, Gothenburg, Sweden; ${ }^{3}$ Department of Research and Development, Region Halland, Halmstad, Sweden; ${ }^{4}$ Institute of Medicine, Sahlgrenska Academy, University of Gothenburg, Gothenburg, Sweden; ${ }^{5}$ University of Lund, Lund, Sweden; ${ }^{6}$ COPD Center, Institute of Medicine, Sahlgrenska University Hospital, University of Gothenburg, Gothenburg, Sweden; ${ }^{7}$ Karolinska Institute, Department of Oncology-Pathology, Stockholm, Sweden; ${ }^{8}$ Research and Development Unit, Stockholms Sjukhem Foundation, Stockholm, Sweden
Correspondence: Ingela Henoch Sahlgrenska Academy at the University of Gothenburg, Institute of Health and Care Sciences, Box 457, Gothenburg SE-405

30 , Sweden

Tel +46317866092

Email ingela.henoch@gu.se
Background: Both single factors and composite measures have been suggested to predict mortality in patients with chronic obstructive pulmonary disease (COPD) and there is a need to analyze the relative importance of each variable.

Objective: To explore the predictors of mortality for patients with COPD in relation to respiratory, cardiac, and malignant causes, as well as all causes of death.

Methods: After merging the Swedish Respiratory Tract Register (SRTR) and the Swedish Cause of Death Register, patients with respiratory, cardiac, and other causes of death were identified. Demographic and clinical variables from the deceased patients' first registration with the SRTR were compared. Three univariable and multivariable Cox proportional hazards regression analyses were conducted for different causes of death, with time from first registration to either death or a fixed end date as dependent variable, and variables regarding demographics, respiration, and comorbidities as independent variables.

Results: In the multivariable Cox models, mortality for patients with all causes of death was predicted by older age 1.79 (CI 1.41, 2.27), lower percentage of predicted forced expiratory volume in 1 second $\left(\mathrm{FEV}_{1} \%\right) 0.99$ (CI 0.98, 0.99), lower saturation 0.92 (CI 0.86, 0.97), worse dyspnea 1.48 (CI 1.26, 1.74) $(\mathrm{p}<0.002$ to $\mathrm{p}<0.001)$, less exercise 0.91 (CI 0.85, 0.98), and heart disease 1.53 (CI 1.06, 2.19) (both $\mathrm{p}<0.05$ ). Mortality for patients with respiratory causes was predicted by higher age 1.67 (CI 1.05, 2.65) $(\mathrm{p}<0.05)$, lower FEV $_{1 \%} 0.98$ (CI 0.97, 0.99), worse dyspnea 2.05 (CI 1.45, 2.90), and a higher number of exacerbations 1.27 (CI 1.11, 1.45) ( $<<0.001$ in all comparisons). For patients with cardiac causes of death, mortality was predicted by lower $\mathrm{FEV}_{1 \%} 0.99$ (CI 0.98, 0.99) $(\mathrm{p}=0.001)$ and lower saturation 0.82 (CI 0.76, 0.89) $(\mathrm{p}<0.001)$, older age $1.46(\mathrm{CI} 1.02,2.09)(\mathrm{p}<0.05)$, and presence of heart disease at first registration 2.06 (CI 1.13, 3.73) $(\mathrm{p}<0.05)$.

Conclusion: Obstruction predicted mortality in all models and dyspnea in two models and needs to be addressed. Comorbidity with heart disease could further worsen the COPD patient's prognosis and should be treated by a multidisciplinary team of professional specialists.

Keywords: chronic obstructive pulmonary disease, COPD, mortality, quality register research

\section{Introduction}

Whereas early stages of chronic obstructive pulmonary disease (COPD) can remain undiagnosed due to a variable or uncharacteristic symptom picture, advanced disease is a leading cause of death worldwide. The death rates from COPD from 1990 to 2015 increased by $11.6 \%$. When age-standardized, there was, however, 
a decrease in death rates from COPD in the high to middle income countries $(-20-22 \%)$, while lower in low income countries $(-1.6 \%)^{1}$

Several single factors related to mortality in COPD patients have been suggested, such as dyspnea, ${ }^{2-4}$ comorbidity (eg, with cardiovascular disease, lung cancer, osteoporosis, and depression ${ }^{2}$ ), number of comorbidities, ${ }^{5}$ body mass index (BMI), ${ }^{3,6}$ percentage of predicted forced expiratory volume in 1 second $\left(\mathrm{FEV}_{1} \%\right)^{2,3,6}$ exercise capacity, ${ }^{2,3,6}$ COPD exacerbations, ${ }^{2,3}$ biomarkers, ${ }^{2,3}$ inspiratory fraction ${ }^{2,3}$ or inspiratory capacity, ${ }^{2}$ and anemia. ${ }^{6}$ Dyspnea has been shown to be a better predictor of 5-year survival than $\mathrm{FEV}_{1} \%{ }^{2}$

To improve the accuracy of predictive factors, efforts have been made to construct predictive indices. The BODE is such a composite measure of severity of COPD, comprising body mass index (BMI), Obstruction, Dyspnea, and Exercise capacity. The instrument has been found to predict both hospitalization ${ }^{2,7}$ and mortality. ${ }^{2,8}$ In a validation study, the BODE predicted mortality better than obstruction as a single measure. ${ }^{9}$ Also, in an evaluation comparing the Global initiative for chronic Obstructive Lung Disease (GOLD) staging, the BODE, and a comorbidity index, the BODE was found to be superior in predicting mortality. ${ }^{10}$

In one study using the BODE, exercise capacity was replaced with peak oxygen uptake $\left(\mathrm{VO}_{2}\right.$ max). It was found that the original BODE was superior. ${ }^{11}$ There are, however, conflicting data: In another study, ${ }^{12} \mathrm{VO}_{2}$ was found to be a better predictor of mortality than the BODE and other composite measures such as the DOSE (which comprises Dyspnea, Obstruction, Smoking, and Exacerbations ${ }^{13}$ ) or ADO (which includes Age, Dyspnea, and Obstruction). ${ }^{14}$

In a large cohort of more than 3000 patients, with more than 1,200 deaths, the ADO index was the best predictor of mortality, followed by the BODE. ${ }^{15}$ Both short-term (6-12 months) and long-term (10 years) survivors were analyzed. Abu Hussein et al $(2014)^{16}$ found that the ADO was an independent predictor of 2-year mortality in three samples of COPD patients from general practice. The ADO index was also found to be a predictor of 3-year mortality in 10 cohorts including more than 13,000 patients. ${ }^{17}$

Puhan et al (2009) $)^{14}$ used both the ADO and a modified form of the BODE and discuss whether the included measurements should be weighted in order to better predict mortality, as there is a covariance between the significant variables. All measures of predictors are highly correlated and therefore composites that use addition (instead of weights, eg, ones based on multivariate estimates) have shortcomings. As an example, Marin et al $(2013)^{15}$ suggested age in the ADO index to be an obvious predictor of death. However, BMI and exercise capacity are also predictors of death, independent of age. ${ }^{15}$ In addition, obstruction and cigarette smoking, scored in the DOSE, are related to each other. ${ }^{6}$ Such findings indicate that there is a need to use multivariate analysis such as Cox regression, in order to exclude interrelated variables and to gain knowledge of the relative importance of each variable.

The aim of this study was to explore the most important predictors of mortality for patients with COPD with general, respiratory, and cardiac causes of death in univariable and multivariable Cox models.

\section{Patients and Methods}

This is a register study of two national registers in Sweden that were merged. The Swedish Respiratory Tract Register (SRTR) was introduced in 2009 and includes an increasing number of clinics for patients with respiratory tract diseases. The coverage of the SRTR of patients with severe and very severe COPD has been estimated to more than $80 \%$. The staff at the different clinics register the data about demographic, disease-, and treatment-related variables for patients with COPD and asthma. Data are either directly registered or are automatically retrieved from medical records. When the register started, registrations were mainly made by pulmonary departments. Registrations from primary care were delayed which explains the long time interval between diagnosis and first registration. The patients with COPD were extracted. At the time of data collection, the SRTR comprised data for 39,830 unique COPD patients, 17,410 men and 22,420 women, 4382 of whom had died. The deceased patients were matched by social security number with individuals registered in the Swedish Cause of Death Register and the two registers were merged. The first registration in the SRTR for all patients in the merged register was identified, ie, the registration at the patient's first visit to the clinic. This was done in order to identify early predictors of death.

Demographic variables from the SRTR comprise age and gender. Patient-related variables concern airway obstruction, measured by $\mathrm{FEV}_{1 \%}$ after bronchodilation if available, otherwise without bronchodilation, as well as oxygen saturation, number of exacerbations, and number 
of hospital admissions due to COPD in the last 12 months, BMI, which was categorized as $<22,22-26,27-30$, and $>30 \mathrm{~kg} / \mathrm{m}^{2}$, and comorbidity. Patient-reported variables comprised smoking, exercise activity, measured by reported number of days with physical activity per week, and functional dyspnea, measured using the modified Medical Research Council (mMRC) Dyspnea Scale. ${ }^{18}$ Health-related quality of life (HRQoL) was measured using the COPD Assessment Test (CAT). ${ }^{19,20}$

From the Swedish Cause of Death Register, the following variables were retrieved: date of death and causes of death, according to the International Classification of Diseases, 10th revision (ICD-10). ${ }^{21}$ In the present study, the primary cause of death for each patient was used. The causes of death that were extracted were respiratory (ICT10 code J00-J99) and cardiac (I00-I99) causes, and malignant neoplasms of respiratory and intrathoracic organs (C30-C39). Causes other than these diagnoses were merged and labeled "other causes."

Descriptive statistics, with means and standard deviations (SDs), calculated for continuous variables were used to describe the sample (Table 1). Categorical variables were presented as numbers and percentages for the total sample and the distribution across the groups, ie, for those with pulmonary causes, cardiac causes, malignant lung neoplasms, and other causes of death. Independent sample $t$-test was used for continuous variables and Fisher's exact test for categorical variables.

Cox proportional hazards regression analysis was used to analyze the relationships between survival time from first registration to date of death, or from first registration to December 31st, 2017, for patients who survived this date. Predictors were analyzed for patients with any cause of death (Table 2), for patients who died from pulmonary causes (Table 3), and for patients who died from cardiac causes (Table 4). Firstly, univariable Cox proportional hazard analysis was performed, using the independent variables age, divided into 10-year intervals, gender, $\mathrm{FEV}_{1} \%$, number of $\mathrm{COPD}$ exacerbations in the last year, number of hospitalizations due to COPD in the last year, BMI, exercise capacity reported as number of days with physical activity per week, smoking status, HRQoL measured by the CAT score, dyspnea measured by mMRC scores, and comorbidity. Secondly, independent variables, with more than 10,000 available registrations for the patients, were selected based on values of $p<0.10$ in the univariable analyses, and entered into the Cox multivariable stepwise regression analysis. The rationale for only including variables with more than 10,000 registered patients was to improve the power of the analysis (10,000 means at least $25 \%$ of all registrations). Age and gender were entered into all analyses, as higher age is known to be a significant predictor of death and women are known to have a higher mean length of life. From the multivariable analyses, only significant predictors with $\mathrm{p}<0.05$ were presented.

The study was approved by the Regional Ethics Committee in Gothenburg in July 2015 (Dnr: 317-15). The patient data in the registers were deidentified.

\section{Results}

Demographic and clinical variables from the first registration of patients in the SRTR dying from different causes are presented in Table 1. Patients with cardiac causes of death had a significantly shorter time between diagnosis and death, and between diagnosis and first registration, compared to patients with respiratory causes (Table 1). The mean time between first registration and death for the diseased patients was 1.6 (SD 1.1) years.

\section{Comparison I: Respiratory and Cardiac Causes of Death}

In the univariable analyses, significantly more women died from respiratory than from cardiac causes (Table 1, two first columns). In general, patients dying from respiratory causes had lower values for a range of respiratory variables, but more rarely had heart diseases, hypertension, and diabetes (Table 1).

\section{Comparison 2: Respiratory Causes and Malignant Respiratory Causes (Lung Cancer)}

Compared to patients dying from malignant respiratory causes, the patients dying from respiratory causes were older, had lower $\mathrm{FEV}_{1} \%$, lower saturation, more exacerbations, and more hospital admissions. They were to a lesser extent still smokers, and they exercised less, and had poorer HRQoL and worse dyspnea. No significant differences were seen in comorbidities.

\section{Comparison 3: Respiratory Causes and Other Causes (Except for Cardiac and Malignant Causes)}

Patients with respiratory causes of death displayed both lower saturation and lower $\mathrm{FEV}_{1} \%$, compared to those dying from other causes. They had more COPD exacerbations and hospitalizations during the last year, as 
Table I Descriptive Statistics on Chronic Obstructive Pulmonary Disease (COPD) Patients ( $=4382$ ) from Their First Registration with the Swedish Respiratory Tract Register (SRTR) to Their Death, by Primary Cause of Death. Differences Between Patients with Respiratory Diseases as Primary Cause of Death, and Patients with Either Cardiac Diseases or Malignant Pulmonary Disease, or Other Cause of Death Were Analyzed Using Independent Samples $t$-Test for Continuous Variables and Fisher's Exact Test for Categorical Variables

\begin{tabular}{|c|c|c|c|c|c|c|c|}
\hline \multirow[b]{2}{*}{ Demographic Variables } & \multicolumn{7}{|c|}{ First Registration of Patients who Later Died of Different Primary Causes } \\
\hline & $\begin{array}{l}\text { Pulmonary } \\
\text { Diseases } \\
(n=686 ; 15.7 \%)\end{array}$ & $\begin{array}{l}\text { Cardiac } \\
\text { Diseases } \\
(n=877 ; \\
20.0 \%)\end{array}$ & p-value & $\begin{array}{l}\text { Malignant } \\
\text { Pulmonary } \\
\text { Diseases }(n=156 \text {; } \\
3.6 \%)\end{array}$ & p-value & $\begin{array}{l}\text { Other } \\
\text { Causes } \\
(n=2,663 ; \\
60.8 \%)\end{array}$ & p-value \\
\hline $\begin{array}{l}\text { Age, mean, yrs (SD) } \\
\text { Gender, } \mathrm{n}(\%) \\
\text { Men } \\
\text { Women }\end{array}$ & $\begin{array}{l}75.9(8.8) \\
290(44.9 \%) \\
356(55.1 \%)\end{array}$ & $\begin{array}{l}76.49(8.84) \\
397(54.8 \%) \\
328(45.2 \%)\end{array}$ & $\begin{array}{l}0.20 \\
<0.001\end{array}$ & $\begin{array}{l}73.3(7.66) \\
66(45.8 \%) \\
78(54.2 \%)\end{array}$ & $\begin{array}{l}0.006 \\
0.85\end{array}$ & $\begin{array}{l}76.3(9.08) \\
1000(48.3 \%) \\
1072(51.7 \%)\end{array}$ & $\begin{array}{l}0.33 \\
0.14\end{array}$ \\
\hline Clinical variables & Mean (SD) & Mean (SD) & & Mean (SD) & & Mean (SD) & \\
\hline $\begin{array}{l}\text { Years between COPD diagnosis and first } \\
\text { registration } \\
\text { Years between first registration and death } \\
\text { Years between COPD diagnosis and death } \\
\text { FEV } \% \\
\text { Saturation } \\
\text { Exacerbations during the last } 12 \text { months } \\
\text { Hospital admissions for COPD in the last } \\
12 \text { months } \\
\text { BMI }\end{array}$ & $\begin{array}{l}6.83(5.67) \\
1.49(1.00) \\
8.25(5.68) \\
36.24(17.22) \\
94.00(4.13) \\
1.61(1.81) \\
0.96(1.35) \\
23.67(5.23)\end{array}$ & $\begin{array}{l}5.35(5.32) \\
1.45(1.04) \\
6.62(5.34) \\
44.89(16.31) \\
94.5(3.43) \\
0.83(1.30) \\
0.60(0.99) \\
25.55(6.09)\end{array}$ & $\begin{array}{l}0.007 \\
0.45 \\
0.003 \\
<0.001 \\
<0.001 \\
<0.001 \\
0.001 \\
<0.001\end{array}$ & $\begin{array}{l}6.52(6.25) \\
1.44(0.90) \\
7.61(6.63) \\
47.90(15.84) \\
95.04(3.23) \\
1.00(1.39) \\
0.56(0.87) \\
24.52(4.96)\end{array}$ & $\begin{array}{l}0.77 \\
0.53 \\
0.56 \\
<0.001 \\
<0.001 \\
0.003 \\
0.069 \\
0.19\end{array}$ & $\begin{array}{l}6.11(7.43) \\
1.72(1.14) \\
8.06(7.50) \\
47.06(17.60) \\
94.56(3.57) \\
0.92(1.50) \\
0.57(1.13) \\
24.92(5.54)\end{array}$ & $\begin{array}{l}0.19 \\
<0.001 \\
0.73 \\
<0.001 \\
<0.001 \\
<0.001 \\
<0.001 \\
<0.001\end{array}$ \\
\hline Patient-reported variables & n (\%) & n (\%) & & n (\%) & & n (\%) & \\
\hline $\begin{array}{l}\text { Smoking } \\
\text { Non-smoking } \\
\text { Have quit smoking >6 months ago } \\
\text { Still smoking }\end{array}$ & $\begin{array}{l}51(11.4 \%) \\
275(61.2 \%) \\
123(27.4 \%)\end{array}$ & $\begin{array}{l}63(13.0 \%) \\
234(48.3 \%) \\
187(38.6 \%)\end{array}$ & 0.024 & $\begin{array}{l}8(8.4 \%) \\
44(46.3 \%) \\
43(45.3 \%)\end{array}$ & 0.003 & $\begin{array}{l}200(15.5 \%) \\
587(45.4 \%) \\
505(39.1 \%)\end{array}$ & 0.043 \\
\hline & Mean (SD) & Mean (SD) & & Mean (SD) & & Mean (SD) & \\
\hline $\begin{array}{l}\text { Exercise activity, days per week } \\
\text { Quality of life according to the CAT score } \\
\text { Functional dyspnea (mMRC) } \\
\text { Number of comorbidities }\end{array}$ & $\begin{array}{l}1.78(2.54) \\
19.89(7.60) \\
3.14(1.09) \\
1.58(1.00)\end{array}$ & $\begin{array}{l}1.96(2.64) \\
17.47(8.38) \\
2.61(1.22) \\
1.79(1.03)\end{array}$ & $\begin{array}{l}0.36 \\
0.001 \\
<0.001 \\
0.001\end{array}$ & $\begin{array}{l}2.50(2.66) \\
16.63(7.74) \\
2.39(1.28) \\
1.49(0.93)\end{array}$ & $\begin{array}{l}0.039 \\
0.006 \\
<0.001 \\
0.45\end{array}$ & $\begin{array}{l}2.14(2.70) \\
17.32(7.69) \\
2.58(1.27) \\
1.66(0.98)\end{array}$ & $\begin{array}{l}0.034 \\
<0.001 \\
<0.001 \\
0.14\end{array}$ \\
\hline Comorbidity & n (\%) & n (\%) & & n (\%) & & n (\%) & \\
\hline Heart disease & $181(62.6 \%)$ & $248(73.8 \%)$ & 0.003 & $29(61.7 \%)$ & 1.00 & $577(71.1 \%)$ & 0.008 \\
\hline Hypertension & $243(71.3 \%)$ & 289 (79.6\%) & 0.011 & $43(70.5 \%)$ & 0.88 & $808(80.3 \%)$ & 0.001 \\
\hline Diabetes & $76(28.8 \%)$ & 115 (42.1\%) & 0.002 & $13(32.5 \%)$ & 0.71 & $286(44.2 \%)$ & $<0.001$ \\
\hline Osteoporosis & $68(31.8 \%)$ & $72(36.0 \%)$ & 0.41 & $5(17.2 \%)$ & 0.13 & $183(38.4 \%)$ & 0.10 \\
\hline Depression & II (44.3\%) & $98(40.0 \%)$ & 0.37 & $15(37.5 \%)$ & 0.50 & $346(52.8 \%)$ & 0.020 \\
\hline Sleep apnea syndrome & $8(4.5 \%)$ & II (6.7\%) & 0.48 & $2(7.4 \%)$ & 0.62 & $27(7.4 \%)$ & 0.26 \\
\hline Alpha I antitrypsin deficiency & $3(1.8 \%)$ & 0 & 0.25 & 0 & 1.00 & $5(1.5 \%)$ & 1.00 \\
\hline Lung neoplasms & $13(6.3 \%)$ & $5(2.9 \%)$ & 0.15 & $12(30.8 \%)$ & $<0.001$ & $38(9.0 \%)$ & 0.28 \\
\hline
\end{tabular}

Abbreviations: BMI, body mass index; CAT, COPD Assessment Test; COPD, chronic obstructive pulmonary disease; $\mathrm{FEV}_{1}$, forced expiratory volume in I second; $\mathrm{FEV}_{1} \%$, percentage of predicted FEV 1 ; mMRC, modified Medical Research Council (Dyspnea Scale); SD, standard deviation.

well as lower BMI. Moreover, they were less physically active, and had lower HRQoL and more severe dyspnea.
Patients with other causes of death were to a larger extent still smokers. They also displayed a higher frequency of heart diseases, hypertension, depression, and diabetes. 
Table 2 Cox Regression, with Time from First Registration to Death for Deceased Patients ( $n=4382$ ), and from First Registration to December 31st, 2017, for Patients Who Were Censored on This Date, as Dependent Variable, and Data from First Registration in the Swedish Respiratory Tract Register (SRTR) as Independent Variables

\begin{tabular}{|c|c|c|}
\hline \multirow[t]{2}{*}{ Independent Variables } & $\begin{array}{l}\text { Univariable Analysis Adjusted for Age and } \\
\text { Gender }\end{array}$ & $\begin{array}{l}\text { Multivariable } \\
\text { Analysis }\end{array}$ \\
\hline & HR (95\% Cl) p-value & HR (95\% Cl) p-value \\
\hline $\begin{array}{l}\text { Age (in 10-year intervals) } \\
\text { Gender ( } 1=\text { man, } 2 \text { = woman })\end{array}$ & $\begin{array}{l}2.25(2.17,2.34)<0.001 \quad(n=39,823) \\
0.81(0.76,0.87)<0.001 \quad(n=39,823)\end{array}$ & $1.79(1.41,2.27)<0.001$ \\
\hline $\begin{array}{l}\text { Clinical variables } \\
\text { Years between diagnosis and first registration } \\
\mathrm{FEV}, \% \\
\text { Saturation } \\
\text { Exacerbations during the last } 12 \text { months } \\
\text { Number of hospitalizations for COPD in the last } 12 \text { months }\end{array}$ & $\begin{array}{l}1.04(1.03,1.05)<0.001 \quad(n=12,058) \\
1.00(1.00,1.00)<0.001 \quad(n=16,385) \\
0.89(0.88,0.89)<0.001 \quad(n=19,483) \\
1.23(1.20,1.26)<0.001 \quad(n=16,291) \\
1.33(1.29,1.37)<0.001 \quad(n=5853)\end{array}$ & $\begin{array}{l}0.99(0.98,0.99)<0.001 \\
0.92(0.86,0.97) 0.001\end{array}$ \\
\hline $\begin{array}{l}\text { BMI categorical variable with four categories } \\
\text { BMI } 22-26 \\
\text { BMI }<22 \\
\text { BMI } 27-30 \\
\text { BMI }>30\end{array}$ & $\begin{array}{l}\text { I (total } n=24,108) \\
\text { I.89 }(1.70,2.10)<0.001 \\
0.86(0.75,0.99) 0.035 \\
0.84(0.73,0.96) 0.009\end{array}$ & $\begin{array}{l}\text { I } \\
\text { I.I8 }(0.78, I .79) 0.43 \\
0.73(0.44, I .21) 0.22 \\
0.35(0.19,0.64) 0.001\end{array}$ \\
\hline $\begin{array}{l}\text { Patient-reported variables } \\
\text { Smoking } \\
\text { Non-smoking } \\
\text { Have quit smoking >6 months ago } \\
\text { Current smoking } \\
\text { Exercise activity, days per week } \\
\text { Quality of life, according to the CAT score } \\
\text { Functional dyspnea (mMRC) }\end{array}$ & $\begin{array}{l}\text { I (total } n=28,672) \\
\text { I.47 }(1.30,1.66)<0.00 \text { I } \\
\text { I.62 }(1.42,1.84)<0.001 \\
0.84(0.83,0.86)<0.001 \quad(n=20,012) \\
\text { I.06 }(1.06,1.07)<0.001 \quad(n=14,779) \\
\text { I.70 }(1.62,1.77)<0.00 \text { I }(n=14,235)\end{array}$ & $0.91(0.85,0.98) 0.012$ \\
\hline $\begin{array}{l}\text { Comorbidity } \\
\text { Heart disease } \\
\text { Hypertension } \\
\text { Diabetes } \\
\text { Osteoporosis } \\
\text { Sleep apnea syndrome } \\
\text { Alpha I antitrypsin deficiency } \\
\text { Lung neoplasms } \\
\text { Depression/anxiety }\end{array}$ & 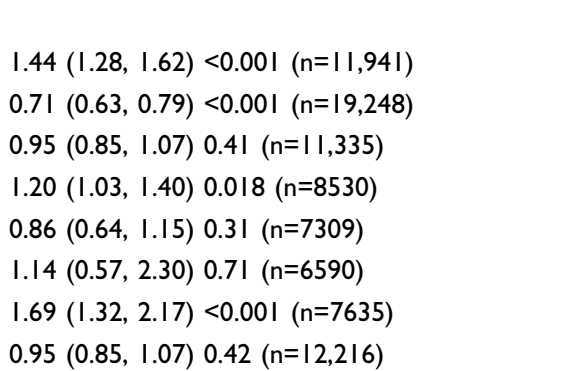 & $1.53(1.06,2.19) 0.022$ \\
\hline
\end{tabular}

Abbreviations: BMI, body mass index; CAT, COPD Assessment Test; $\mathrm{Cl}$, confidence interval; COPD, chronic obstructive pulmonary disease; FEV ${ }_{1}$, forced expiratory volume in I second; $\mathrm{FEV}_{1} \%$, percentage of predicted $\mathrm{FEV}_{1}$; HR, hazard ratio; mMRC, modified Medical Research Council (Dyspnea Scale).

\section{Cox Regression Models - All Causes of Death}

In the initial univariable Cox regression, all causes of death were investigated, where time from first registration was chosen as dependent variable and variables regarding demographics, respiration, HRQoL, and comorbidities were entered as independent variables. In this model, mortality was predicted by most of the inserted variables, except for some comorbidities (Table 2).
In the multivariable, stepwise Cox regression analysis, all variables that became predictors based on $\mathrm{p}<0.10$ and with more than 10,000 registered patients in the univariable analyses, were entered as independent variables. Older age, lower $\mathrm{FEV}_{1} \%$, lower saturation, lower number of days with exercise per week, worse dyspnea, low BMI, and heart disease remained significant in the multivariable analysis (Table 2). 
Table 3 Cox Regression, with Time from First Registration to Death for Patients Who Died from Respiratory Causes, and from First Registration to December 3Ist, 2017, for Patients Who Were Censored on That Date, as Dependent Variable, and Data from First Registration as Independent Variables.*

\begin{tabular}{|c|c|c|}
\hline Independent Variables & $\begin{array}{l}\text { Univariable Analysis Adjusted for Age and } \\
\text { Gender }\end{array}$ & $\begin{array}{l}\text { Multivariable } \\
\text { Analysis }\end{array}$ \\
\hline & HR (95\% Cl) p-value & HR (95\% Cl) p-value \\
\hline Age (in 10-year intervals) & $2.14(1.96,2.34)<0.001 \quad(n=39,818)$ & $1.67(1.05,2.65) 0.029$ \\
\hline $\begin{array}{l}\text { Gender } \\
\text { Male } \\
\text { Female }\end{array}$ & $\begin{array}{l}I(n=39,818) \\
0.95(0.82,1.11) 0.95\end{array}$ & \\
\hline $\begin{array}{l}\text { Clinical variables } \\
\text { Years between diagnosis and first registration } \\
\mathrm{FEV}, \% \\
\text { Saturation } \\
\text { Exacerbations during the last } 12 \text { months } \\
\text { Number of hospitalizations for COPD in the last } 12 \text { months }\end{array}$ & $\begin{array}{l}1.05(1.03,1.07)<0.001 \quad(n=12,046) \\
1.00(1.00,1.00) 0.003 \quad(n=16,378) \\
0.86(0.85,0.88)<0.001 \quad(n=19,468) \\
1.38(1.32,1.43)<0.001 \quad(n=16,280) \\
1.40(1.33,1.47)<0.001 \quad(n=5848)\end{array}$ & $\begin{array}{l}0.98(0.97,0.99) 0.00 \mathrm{I} \\
\mathrm{I} .27(\mathrm{I} . \mathrm{II}, \mathrm{I} .45)<0.00 \mathrm{I}\end{array}$ \\
\hline $\begin{array}{l}\text { BMI categorical variable with four categories } \\
\text { BMI } 22-26 \\
\text { BMI }<22 \\
\text { BMI } 27-30 \\
\text { BMI }>30\end{array}$ & $\begin{array}{l}I(n=24,093) \\
2.1 I(I .67,2.67)<0.00 I \\
0.67(0.48,0.95) 0.023 \\
0.55(0.40,0.78) 0.001\end{array}$ & $\begin{array}{l}\text { I } \\
\text { I.52 }(0.68,3.36) 0.3 \text { I } \\
\text { I.00 }(0.37,2.73) 0.99 \\
0.1 I(0.01,0.97) 0.047\end{array}$ \\
\hline $\begin{array}{l}\text { Patient-reported variables } \\
\text { Smoking } \\
\text { Non-smoking } \\
\text { Have quit smoking >6 months ago } \\
\text { Current smoking } \\
\text { Exercise activity, days per week } \\
\text { Quality of life, according to the CAT score } \\
\text { Functional dyspnea (mMRC) }\end{array}$ & $\begin{array}{l}I(n=28,653) \\
2.23(1.66,3.01)<0.001 \\
1.41(1.01,1.97) 0.043 \\
0.81(0.77,0.85)<0.001 \quad(n=19,998) \\
1.10(1.08,1.12)<0.001 \quad(n=14,768) \\
2.40(2.15,2.77)<0.001 \quad(n=14,223)\end{array}$ & $2.05(1.45,2.90)<0.001$ \\
\hline $\begin{array}{l}\text { Comorbidity } \\
\text { Heart disease } \\
\text { Hypertension } \\
\text { Diabetes } \\
\text { Osteoporosis } \\
\text { Sleep apnea syndrome } \\
\text { Alpha I antitrypsin deficiency } \\
\text { Lung neoplasms } \\
\text { Depression/anxiety }\end{array}$ & $\begin{array}{l}1.00(0.77,1.28) 0.98 \quad(n=11,937) \\
0.47(0.37,0.60)<0.001 \quad(n=19,244) \\
0.55(0.42,0.72)<0.001 \quad(n=11,323) \\
0.89(0.65,1.22) 0.46 \quad(n=8516) \\
0.58(0.28,1.18) 0.14 \quad(n=7303) \\
1.64(0.52,5.17) 0.39 \quad(n=6564) \\
1.20(0.68,2.10) 0.54 \quad(n=7627) \\
0.78(0.61,1.00) 0.049 \quad(n=12,209)\end{array}$ & \\
\hline
\end{tabular}

Notes: *Malignant pulmonary diseases were studied in an univariable comparison with respiratory diseases in Table 4, but were not entered in the multivariable analyses as they constituted only $0.4 \%$ of all patients.

Abbreviations: BMI, body mass index; CAT, COPD Assessment Test; $\mathrm{Cl}$, confidence interval; COPD, chronic obstructive pulmonary disease; FEV ${ }_{1}$, forced expiratory volume in I second; FEV $\%$, percentage of predicted FEV ; HR, hazard ratio; mMRC, modified Medical Research Council (Dyspnea Scale).

\section{Cox Regression Models - Respiratory Causes of Death}

In our second main model, the variable "respiratory causes of death" was studied. Time from first registration was entered as dependent variable, and variables regarding demographics, respiration, HRQoL, and comorbidities were entered into a univariable Cox regression model as independent variables (see Table 3). In the multivariable stepwise Cox regression analysis including variables from the univariable analysis with more than 10,000 registrations and $\mathrm{p}<0.10$, older age, lower 
Table 4 Cox Regression, with Time from First Registration to Death for Patients Who Died from Cardiac Causes, and with Time from First Registration to December 3Ist, 2017, for Patients Who Were Censored on That Date, as Dependent Variable, and Data from First Registration as Independent Variables

\begin{tabular}{|c|c|c|}
\hline \multirow[t]{2}{*}{ Independent Variables } & $\begin{array}{l}\text { Univariable Analysis Adjusted for Age and } \\
\text { Gender }\end{array}$ & $\begin{array}{l}\text { Multivariable } \\
\text { Analysis }\end{array}$ \\
\hline & HR (95\% Cl) p-value & HR (95\% Cl) p-value \\
\hline Age (in 10-year intervals) & $2.32(2.13,2.53)<0.001 \quad(n=39,821)$ & $\mathrm{I} .46(\mathrm{I} .02,2.09) 0.04 \mathrm{I}$ \\
\hline $\begin{array}{l}\text { Gender } \\
\text { Male } \\
\text { Female }\end{array}$ & $\begin{array}{l}I(n=39,82 I) \\
0.64(0.55,0.74)<0.00 I\end{array}$ & \\
\hline $\begin{array}{l}\text { Clinical variables } \\
\text { Years between diagnosis and first registration } \\
\mathrm{FEV}, \% \\
\text { Saturation } \\
\text { Exacerbations during the last } 12 \text { months } \\
\text { Number of hospitalizations for COPD in the last } 12 \text { months }\end{array}$ & $\begin{array}{l}1.02(1.00,1.04) 0.10 \quad(n=12,057) \\
1.00(1.00,1.00) 0.037 \quad(n=16,385) \\
0.90(0.88,0.92)<0.001 \quad(n=19,483) \\
1.13(1.06,1.22)<0.001 \quad(n=16,291) \\
1.28(1.19,1.38)<0.001 \quad(n=5853)\end{array}$ & $\begin{array}{l}0.99(0.98,0.99) 0.001 \\
0.82(0.76,0.89)<0.00 \text { I }\end{array}$ \\
\hline $\begin{array}{l}\text { BMI categorical variable with four categories } \\
\text { BMI } 22-26 \\
\text { BMI }<22 \\
\text { BMI } 27-30 \\
\text { BMI }>30\end{array}$ & $\begin{array}{l}I(n=24,107) \\
2.00(1.57,2.55)<0.001 \\
1.09(0.82,1.45) 0.57 \\
I .19(0.90,1.56) 0.22\end{array}$ & \\
\hline $\begin{array}{l}\text { Patient-reported variables } \\
\text { Smoking } \\
\text { Non-smoking } \\
\text { Have quit smoking > } 6 \text { months ago } \\
\text { Current smoking } \\
\text { Exercise activity, days per week } \\
\text { Quality of life, according to the CAT score } \\
\text { Functional dyspnea (mMRC) }\end{array}$ & $\begin{array}{l}\text { I }(n=28,67 I) \\
\text { I.50 }(I .14,1.99) 0.004 \\
\text { I.78 }(1.33,2.38)<0.001 \\
0.83(0.80,0.87)<0.001 \quad(n=20,011) \\
1.06(1.04,1.08)<0.001 \quad(n=14,779) \\
1.59(1.46,1.74)<0.001 \quad(n=14,235)\end{array}$ & \\
\hline $\begin{array}{l}\text { Comorbidity } \\
\text { Heart disease } \\
\text { Hypertension } \\
\text { Diabetes } \\
\text { Osteoporosis } \\
\text { Sleep apnea syndrome } \\
\text { Alpha I antitrypsin deficiency } \\
\text { Lung neoplasms } \\
\text { Depression/anxiety }\end{array}$ & 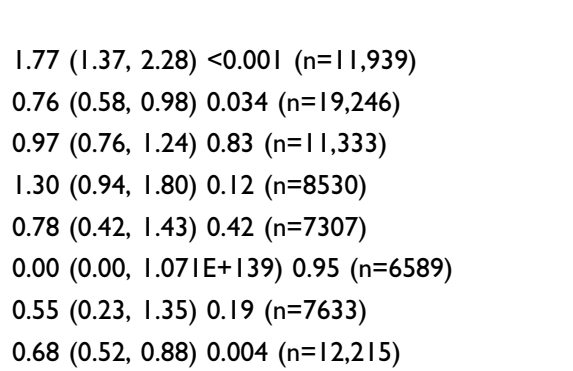 & $2.06(1.13,3.73) 0.018$ \\
\hline
\end{tabular}

Abbreviations: BMI, body mass index; CAT, COPD Assessment Test; $\mathrm{Cl}$, confidence interval; COPD, chronic obstructive pulmonary disease; FEV ${ }_{1}$, forced expiratory volume in I second; FEV $\%$, percentage of predicted $\mathrm{FEV}_{1}$; HR, hazard ratio; mMRC, modified Medical Research Council (Dyspnea Scale).

$\mathrm{FEV}_{1} \%$, a higher number of exacerbations, lower BMI, and worse dyspnea became significant predictors of mortality.

\section{Cox Regression Models - Cardiac Causes of Death}

In a Cox regression model regarding cardiac causes of death, with time from first registration as dependent variable, and including the same independent variables as in the other models, all variables except for some comorbidities became univariable predictors (Table 4). In the multivariable stepwise Cox regression analysis, all variables with more than 10,000 registrations were included based on $p<0.10$. In this model, older age, lower $\mathrm{FEV}_{1 \%}$ and saturation, and the presence of heart disease at first registration predicted mortality with cardiac causes. 


\section{Discussion}

The present study shows that in all all-cause, respiratory, and cardiac mortality, the respiratory variables were predictors of mortality, with some variations. Older age and low $\mathrm{FEV}_{1 \%}$ predicted mortality in all models. BMI and dyspnea remained significant in analyses of all causes and respiratory causes of death, whereas low saturation and presence of heart diseases were predictors of all causes and cardiac causes of death. Exacerbations predicted mortality of respiratory causes. This together indicates that subjective and objective variables as well as comorbidities need to be considered when predicting mortality.

A large number of respiratory and non-respiratory variables have been suggested as predictors of mortality. This is logical as COPD in the early stages mainly produces respiratory problems, ${ }^{22}$ more advanced stages are associated with various comorbidities, eg, heart failure, loss of weight/ cachexia, osteoporosis, and depression. However, the present study shows that the registered reductions in respiratory function remain as predictors of mortality more than one year later. Among respiratory variables, dyspnoea, $\mathrm{FEV}_{1} \%$, saturation, and exacerbations of COPD are well-known predictors of poor prognosis, ${ }^{2-4,6}$ which is well in agreement with our findings. In addition, hospitalization, a strong surrogate variable mainly associated with COPD exacerbations and dyspnoea, is a wellknown risk factor. ${ }^{23-25}$ Moreover, comorbidities including, eg, heart failure, low BMI, and depression have all been associated with an increased risk of dying from COPD. ${ }^{26-29}$ In order to obtain a higher precision, composites such as the BODE and ADO have been created, with proved predictive value in certain studies. $^{2,7,8,14,15,17,27}$

Many of the commonly suggested variables covary within the same patient. A person with severe COPD is likely to present with dyspnoea, as well as insufficient saturation, low $\mathrm{FEV}_{1} \%$, and episodes of exacerbations, and so on. Each of these variables is a predictor of prognosis at a group level, but with insufficient precision to accurately predict survival in the individual patient. A drawback of these composites is, however, that various variables have been used in an additive way, despite the strong correlation between the included items. Although such composites may obviously strengthen the prognostic capacity to some degree,,$^{3,9,29,30}$ they do not overcome the problem of covariation. Puhan et $\mathrm{al}^{14}$ argue that health care would benefit from more specific predictors of mortality in order to determine thresholds where more, or less, treatment should be proposed to have an acceptable risk- benefit ratio. However, no reliable thresholds have been determined so far. A multivariable approach, eg, using Cox proportional hazards analyses, may be a step in that direction in order to determine the relative importance of different variables. $\mathrm{Eg}$, in the present study the hazards ratio for dyspnea was higher than the hazards ratio for $\mathrm{FEV}_{1} \%$ in the all cause and respiratory causes of mortality, which put emphasis on the relative importance of the subjective experience as a complement to objective measurements. This is in line with new GOLD grading system that has been supplemented with symptom assessment and history of exacerbations. ${ }^{31,32}$ Although the present study was not designed to propose thresholds where more, or less, treatment should be introduced to have an acceptable risk-benefit ratio, we argue that the subjective experience is of great importance as a predictor, but also as a basis for an acceptable quality of life at the end of life. All predictors are developed on population level. Still, predictors derived from multivariable regression models have a higher precision also in the individual case, as there is a high co-variation between significant variables identified in univariable regression models. The multivariable models take this co-variation into account.

Others, such as Celli et al, ${ }^{6}$ argue that greater efforts should be made to analyze respiratory causes of mortality from non-respiratory causes, in order to better understand the effects of COPD on mortality. This is in line with our findings, as variables that were independent predictors of mortality from all causes of death differed from those predicting death from respiratory causes. This is clinically important, as COPD in the early stages is mainly a lung disease, whereas in the late stages, it is intimately associated with comorbidities that also contribute to shortened survival. In contrast to composite indices as the BODE, ${ }^{6}$ DOSE, ${ }^{13}$ and $\mathrm{ADO}^{17}$, the present study take different causes of death in consideration. As the models in the present study resulted in some differences in predictors, it is important to explore the importance of cause of death.

In the three Cox regression analyses in the present study, some predictors were generic and obvious, such as age, while others varied somewhat. In the first multivariable analysis with all causes of death, age, $\mathrm{FEV}_{1} \%$, saturation, exercise activity, dyspnea, low BMI, and presence of heart disease became significant predictors. These predictors indicate that the effect of the COPD disease was obvious, which is in line with the composite measures, the BODE, ${ }^{6}$ DOSE, ${ }^{13}$ and ADO, ${ }^{17}$ which all include dyspnea 
and obstruction (the ADO also includes age). The BODE ${ }^{6}$ also includes exercise and BMI, as in our study. DOSE ${ }^{13}$ includes dyspnea, obstruction, smoking, and number of exacerbations. However, in our study smoking does not predict mortality. In the univariable analysis, smoking is somewhat contradictive in that those who had quit smoking had higher mortality than those who were still smokers. Possibly, this is a vulnerable population and those who had quit smoking had quitted due to more severe illness. In our data, heart disease also became a significant predictor of mortality. The importance of comorbidities, especially heart disease, for COPD patients' mortality has been demonstrated by others, ${ }^{23,26}$ which indicates that frail patients with multiple diseases need to be professionally treated. Composite indices, as well as the results from the present study, indicate important factors for predicting mortality, which could be used in clinical practice to initiate discussions with patients and families of individual preferences of care. As there could be individual and cultural differences in expectations of care, healthcare professionals need to adapt such discussions accordingly. The independent variables in the present study were collected at the patients' first registration in the register, which resulted in a prediction of mortality at an early stage. Early reliable predictors might be a basis to promote early changes in treatment and care, which could be beneficial for the patient.

Not surprisingly, in the second Cox regression analysis concerning respiratory cause of death, COPD-related variables were shown to predict mortality in the multivariable analysis, ie, $\mathrm{FEV}_{1} \%$, number of exacerbations in the last year, lower BMI, and dyspnea. This points to the usefulness of the DOSE, ${ }^{13}$ which includes dyspnea, obstruction, smoking, and number of exacerbations. However, in our study, smoking does not predict mortality. The importance of dyspnea as a predictor of mortality, also when controlling for age, smoking, and lung function, has been acknowledged by others. ${ }^{33,34}$ In the comparisons between patients with different causes of death, presented in Table 1, patients who died from respiratory causes had significantly worse dyspnea and HRQoL than patients dying from other causes. This emphasizes the importance of treating dyspnea and there are studies showing that, eg, pulmonary rehabilitation has effects on surrogate variables such as dyspnea and exercise capacity. ${ }^{6,35}$ An interesting finding in our population was that patients dying from respiratory causes had significantly worse dyspnea (mMRC scores) compared to patients dying from lung cancer, despite the fact that lung cancer patients often are smokers/previous smokers.

In the third Cox regression analysis, conducted in patients with a cardiac cause of death, higher age, lower $\mathrm{FEV}_{1} \%$, and lower saturation, as well as presence of heart disease at first registration remained significant in the multivariable analysis. This indicates that although these patients died from a cardiac cause of death, the COPD disease still had a significant impact on them. In COPD patients with heart disease, the COPD treatment therefore needs to be continued.

Both clinicians and patients need prognostic information. From a health-care point of view, the identification of prognostic markers or composites is of interest, as such information may facilitate tailored treatments. The finding of low $\mathrm{FEV}_{1 \%}$ as a significant predictor of mortality in all-cause, respiratory, and cardiac mortality, and dyspnea in all-cause and respiratory mortality, indicates that dyspnea needs to be targeted, regardless of the primary diagnosis. Recent efforts have been made to acknowledge the need to address dyspnea in both COPD and heart disease simultaneously. ${ }^{36}$

The use of quality registers, such as the SRTR, has the potential to generate knowledge about the effect of interventions on large populations. ${ }^{37}$ Thus, although not allcompassing in either geographical coverage or inclusion in every health-care center, the register still provides valuable information in the context of real patient encounters. A limitation of this study is that the registration in the SRTR was not complete for all patients, resulting in missing values in the multivariable Cox proportional hazards regression analysis. In order to further improve predictions of mortality, other variables could have been included, eg biomarkers or data on inspiratory function. Another limitation is that analysis of interaction of the variables were not executed.

To summarize, our study has confirmed the predictive value of age and $\mathrm{FEV}_{1 \%}$ in all-cause, respiratory, and cardiac mortality and dyspnea in all-cause and respiratory mortality. Moreover, the time point at which potential predictors are registered will affect the outcome. Certain respiratory variables, such as $\mathrm{FEV}_{1 \%}$ or saturation, may be present and registered early, whereas variables such as osteoporosis or low BMI develop later in the trajectory. Moreover, for the patients who died from a cardiac cause of death, heart disease at the first registration was a strong predictor of mortality. Therefore, there is no simple way to decide on an optimal model of decision making as regards prognosis and treatment, as several models are possible. 
The clinician should bear in mind that a reasonable prognosis can be made from those variables available in a certain clinical situation.

\section{Disclosure}

The authors report no conflicts of interest in this work.

\section{References}

1. Soriano JB, Abajobir AA, Hassen Abate K, et al. for GBD 2015 chronic respiratory disease collaborators. Global, regional, and national deaths, prevalence, disability-adjusted life years, and years lived with disability for chronic obstructive pulmonary disease and asthma, 1990-2015: a systematic analysis for the global burden of disease study 2015. Lancet Respir Med. 2017;5(9):691-706.

2. Papaioannou AI, Loukides S, Gourgoulianis KI, Kostikas K. Global assessment of the COPD patient: time to look beyond FEV1? Respir Med. 2009;103(5):650-660. doi:10.1016/j.rmed.2009.01.001

3. Celli BR. Predictors of mortality in COPD. Respir Med. 2010;104 (6):773-779. doi:10.1016/j.rmed.2009.12.017

4. Motegi T, Jones RC, Ishii T, et al. A comparison of three multidimensional indices of COPD severity as predictors of future exacerbations. Int J Chron Obstruct Pulmon Dis. 2013;8:259-271. doi:10.2147/COPD.S42769

5. Eroglu SA, Gunen H, Yakar HI, Yildiz E, Kavas M, Duman D. Influence of comorbidities in long-term survival of chronic obstructive pulmonary disease patients. $J$ Thorac Dis. 2019;11 (4):1379-1386. doi:10.21037/jtd.2019.03.78

6. Celli BR, Cote CG, Lareau SC, Meek PM. Predictors of Survival in COPD: more than just the FEV1. Respir Med. 2008;102(Suppl 1): S27-S35. doi:10.1016/S0954-6111(08)70005-2

7. Ong KC, Earnest A, Lu SJ. A multidimensional grading system (BODE index) as predictor of hospitalization for COPD. Chest. 2005;128(6):3810-3816. doi:10.1378/chest.128.6.3810

8. Soler-Cataluna JJ, Martinez-Garcia MA, Sanchez LS, Tordera MP, Sanchez PR. Severe exacerbations and BODE index: two independent risk factors for death in male COPD patients. Respir Med. 2009;103(5):692-699. doi:10.1016/j.rmed.2008.12.005

9. Celli BR, Cote CG, Marin JM, et al. The body-mass index, airflow obstruction, dyspnea, and exercise capacity index in chronic obstructive pulmonary disease. $N$ Engl J Med. 2004;350(10):1005-1012. doi:10.1056/NEJMoa021322

10. de Torres JP, Casanova C, Marin JM, et al. Prognostic evaluation of COPD patients: GOLD 2011 versus BODE and the COPD comorbidity index COTE. Thorax. 2014;69(9):799-804. doi:10.1136/thoraxjnl-2014-205770

11. Cote CG, Pinto-Plata VM, Marin JM, Nekach H, Dordelly LJ, Celli BR. The modified BODE index: validation with mortality in COPD. Eur Respir J. 2008;32(5):1269-1274. doi:10.1183/ 09031936.00138507

12. Oga T, Tsukino M, Hajiro T, Ikeda A, Nishimura K. Predictive properties of different multidimensional staging systems in patients with chronic obstructive pulmonary disease. Int $J$ Chron Obstruct Pulmon Dis. 2011;6:521-526. doi:10.2147/COPD.S24420

13. Sundh J, Janson C, Lisspers K, Stallberg B, Montgomery S. The Dyspnoea, Obstruction, Smoking, Exacerbation (DOSE) index is predictive of mortality in COPD. Primary Care Respir j. 2012;21 (3):295-301. doi:10.4104/pcrj.2012.00054

14. Puhan MA, Garcia-Aymerich J, Frey M, et al. Expansion of the prognostic assessment of patients with chronic obstructive pulmonary disease: the updated BODE index and the ADO index. Lancet. 2009;374(9691):704-711. doi:10.1016/S0140-6736(09)61301-5
15. Marin JM, Alfageme I, Almagro P, et al. Multicomponent indices to predict survival in COPD: the COCOMICS study. Eur Respir J. 2013;42(2):323-332. doi:10.1183/09031936.00121012

16. Abu Hussein N, Ter Riet G, Schoenenberger L, et al. The ADO index as a predictor of two-year mortality in general practice-based chronic obstructive pulmonary disease cohorts. Respiration. 2014;88 (3):208-214. doi:10.1159/000363770

17. Puhan MA, Hansel NN, Sobradillo P, et al. Large-scale international validation of the ADO index in subjects with COPD: an individual subject data analysis of 10 cohorts. BMJ Open. 2012;2(6):e002152. doi:10.1136/bmjopen-2012-002152

18. Bestall JC, Paul EA, Garrod R, Garnham R, Jones PW, Wedzicha JA. Usefulness of the Medical Research Council (MRC) dyspnoea scale as a measure of disability in patients with chronic obstructive pulmonary disease. Thorax. 1999;54(7):581-586. doi:10.1136/ thx.54.7.581

19. Jones PW, Harding G, Berry P, Wiklund I, Chen WH, Kline Leidy N. Development and first validation of the COPD Assessment Test. Eur Respir J. 2009;34(3):648-654. doi:10.1183/09031936.00102509

20. Becker C, Schafer J, Carvalho LL, Vitiello IP, da Silva AL. CAT correlates positively with respiratory rate and is a significant predictor of the impact of COPD on daily life of patients: a cross sectional study. Multidiscip Respir Med. 2014;9(1):47. doi:10.1186/2049-6958$9-47$

21. WHO. International Classification of Diseases (ICD); 2010. Available from: https://icd.who.int/browse10/2019/en. Accessed 3 May, 2020.

22. Maltais F, Dennis N, Chan CK. Rationale for earlier treatment in COPD: a systematic review of published literature in mild-tomoderate COPD. COPD. 2013;10(1):79-103. doi:10.3109/154125 55.2012.719048

23. Almagro P, Calbo E, Ochoa de Echaguen A, et al. Mortality after hospitalization for COPD. Chest. 2002;121(5):1441-1448. doi:10.1378/chest.121.5.1441

24. Esteban C, Quintana JM, Aburto M, et al. Predictors of mortality in patients with stable COPD. J Gen Intern Med. 2008;23(11):1829-1834. doi:10.1007/s11606-008-0783-x

25. Garcia-Sanz MT, Canive-Gomez JC, Senin-Rial L, et al. One-year and long-term mortality in patients hospitalized for chronic obstructive pulmonary disease. $J$ Thorac Dis. 2017;9(3):636-645. doi: $10.21037 /$ jtd.2017.03.34

26. Kaszuba E, Odeberg H, Rastam L, Halling A. Impact of heart failure and other comorbidities on mortality in patients with chronic obstructive pulmonary disease: a register-based, prospective cohort study. BMC Fam Pract. 2018;19(1):178. doi:10.1186/s12875-018-0865-8

27. Prudente R, Franco EAT, Mesquita CB, Ferrari R, de Godoy I, Tanni SE. Predictors of mortality in patients with COPD after 9 years. Int J Chron Obstruct Pulmon Dis. 2018;13:3389-3398. doi:10.2147/COPD.S174665

28. de Voogd JN, Wempe JB, Koeter GH, et al. Depressive symptoms as predictors of mortality in patients with COPD. Chest. 2009;135 (3):619-625. doi:10.1378/chest.08-0078

29. Sin DD, Anthonisen NR, Soriano JB, Agusti AG. Mortality in COPD: role of comorbidities. Eur Respir J. 2006;28(6):1245-1257. doi:10.1183/09031936.00133805

30. Gelberg J, McIvor RA. Overcoming gaps in the management of chronic obstructive pulmonary disease in older patients: new insights. Drugs Aging. 2010;27(5):367-375. doi:10.2165/11535220000000000-00000

31. Faner R, Noell G, Ramon Badia J, López-Giraldo A, Bakke P, Silverman EK. Distribution, temporal stability and association with all-cause mortality of the 2017 GOLD groups in the ECLIPSE cohort. Respir Med. 2018;141:14-19. doi:10.1016/j.rmed.2018. 06.015

32. Çolak Y, Afzal S, Marott JL, et al. Prognosis of COPD depends on severity of exacerbation history: a population-based analysis. Respir Med. 2019;155:141-147. doi:10.1016/j.rmed.2019.07.021 
33. Pesola GR, Ahsan H. Dyspnea as an independent predictor of mortality. Clin Respir J. 2016;10(2):142-152. doi:10.1111/crj.12191

34. Ahmed T, Steward JA, O'Mahony MS. Dyspnoea and mortality in older people in the community: a 10-year follow-up. Age Ageing. 2012;41(4):545-549. doi:10.1093/ageing/afs049

35. McCarthy B, Casey D, Devane D, Murphy K, Murphy E, Lacasse Y. Pulmonary rehabilitation for chronic obstructive pulmonary disease. Cochrane Database Syst Rev. 2015;2:CD003793. doi:10.1002/ 14651858.CD003793

36. Man WD, Chowdhury F, Taylor RS, et al. Building consensus for provision of breathlessness rehabilitation for patients with chronic obstructive pulmonary disease and chronic heart failure. Chron Respir Dis. 2016;13(3):229-239. doi:10.1177/1479972316642363
37. Bohlin I. Evidence-based desicion-making in a scientific society. About the evidence movement's origin, dispersion and limits. [Swedish: evidensbaserat beslutsfattande $i$ ett vetenskapligt samhälle. Om evidensrörelsens ursprung, utbredning och gränser]. In: Bohlin I, Sager M, editors. The Many Faces of the Evidence. Evidence-Based Practice in Practice. [Swedish: Evidensens Många Ansikten. Evidensbaserad Praktik I Praktiken]. Lund: Arkiv förlag; 2011:31-68.

\section{Publish your work in this journal}

The International Journal of COPD is an international, peer-reviewed journal of therapeutics and pharmacology focusing on concise rapid reporting of clinical studies and reviews in COPD. Special focus is given to the pathophysiological processes underlying the disease, intervention programs, patient focused education, and self management protocols. This journal is indexed on PubMed Central, MedLine and CAS. The manuscript management system is completely online and includes a very quick and fair peer-review system, which is all easy to use. Visit http://www.dovepress.com/testimonials.php to read real quotes from published authors. 